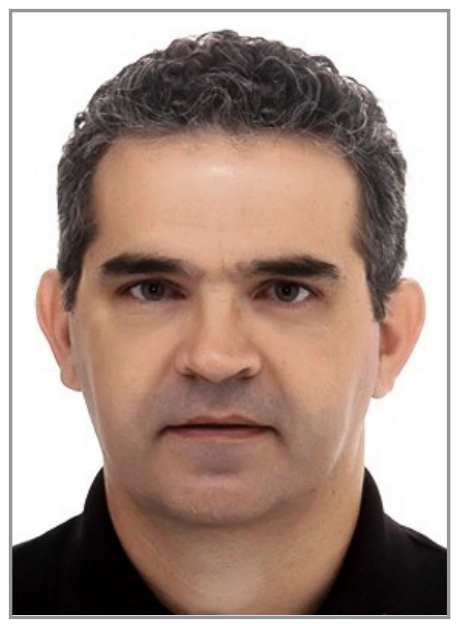

\title{
Analytical Demands for Residues and Contaminants in Food Products
}

\author{
Renato Zanella \\ Full Professor \\ Laboratory for Pesticide Residue Analysis (LARP) \\ Chromatography and Mass Spectrometry Research Group (CPCEM) \\ Department of Chemistry, Federal University of Santa Maria \\ Santa Maria, RS, Brazil \\ renato.zanella@ufsm.br
}

Food marketing increasingly requires the rapid and comprehensive analyses of residues and contaminants, particularly pesticides, veterinary drugs, mycotoxins and heavy metals, in fresh foods as well as in processed products. To safeguard the well-being of consumers, good production practices and adequate analytical control are crucial to enable food producers to gain access to more markets, contributing to economic development. Today, consumers have the right to expect the foods they consume are accessible, safe and of high quality.

Food safety and quality are topics of growing interest to consumers, government agencies and producers. Controlling food residues and contaminants requires broad and sensitive multiresidue methods to confirm their occurrence with confidence and at very low concentration levels, combining modern sample preparation techniques with quick and cost-effective determinations. Residues are organic compounds that appear in food because of their use during production or storage, such as pesticides and veterinary drugs. Contaminants are compounds generated naturally during production, storage or food processing, such as mycotoxins and marine biotoxins, or by anthropogenic activities, such as dioxins and polychlorinated biphenyls. Multiresidue methods are necessary in national food regulatory control systems because they allow food inspection and food safety management to prevent or minimize food safety risks. For prohibited compounds or their metabolites, minimum required performance limits are established to show the levels the analytical methods need to achieve. The complexity and variety of matrix composition of food samples, low concentration of analytes and low required maximum residues levels make it difficult to simultaneously analyse several classes of compounds at the same time. The development of generic extraction procedures combined with multiresidue determination can reduce the number of analyses per sample, saving time and costs. Strategies are needed that update the methods to include newly identified compounds in the validated methods.

The development of multiresidue methods including analytes of current interest allows us to ascertain the quality of food, indicating whether Good Production Practices have been observed along the food production chain, giving greater consumer confidence on the risks of consuming a particular food. The environment and inputs used in the production of food products can also be important sources of contamination, especially metals and persistent organic compounds, and these must be analyzed at very low concentration levels that require modern analysis techniques. The establishment of fast, efficient and comprehensive methods, including the use of different complementary analysis techniques, requires a unified sample preparation step that allows a wide range of compounds to be determined. Analytical methods have a role in assuring food safety of food products. Food safety is the absence or acceptable levels of chemical, physical or microbiological hazards in food that may harm the health of consumers. 
Prohibited additives and counterfeiting harm consumer health and cause serious commercial damage. Also important are the problems of products formed during processing, such as acrylamide, the presence of allergens, prohibited preservatives and mycotoxins. Another important source of contamination are the materials that are already in contact with food or intended to be brought into contact with food. These materials can transfer their constituents to the food products. Food comes into contact with many materials, such as machinery to process food, packaging materials and kitchenware, during its production, processing, storage, distribution, preparation and serving for consumption. Food contact materials should be sufficiently inert to avoid their constituents influencing food quality, and in the process affecting consumer health. To ensure the safety of these materials, appropriate analytical methods are required to investigate the possible contaminants released into the food products. Even very low concentrations of released compounds can be harmful to human health and can negatively influence food quality, changing food composition, taste and odour in an unacceptable way.

The use of new sorbents to obtain cleaner extracts even for very complex food matrices open the possibility of simplifying the sample preparation step for residues and contaminants analyses. In general, highly sensitive and selective gas or liquid chromatography with tandem mass spectrometry using a triple quadrupole analyzer are used for this kind of analyses. To screen non-target and unknown compounds, chromatographic techniques coupled with high-resolution mass spectrometry have been used, generating much information that can be important for food safety.

Considering the current needs for establishing efficient analytical methods for determining residues and contaminants to guarantee food safety, the challenge is to improve the available methods in order to achieve the requirements established by legislation, reducing cost and time even in the case of very complex matrices and difficult compounds. The use of suitable methods and training people with experience in developing and applying efficient analytical methods are important and make analyses results much more reliable. 